\title{
Study of the temperature distribution in Si nanowires under microscopic laser beam excitation
}

\author{
J. Anaya · A. Torres · A. Martín-Martín · J. Souto • \\ J. Jiménez $\cdot$ A. Rodríguez $\cdot$ T. Rodríguez
}

\begin{abstract}
The use of laser beams as excitation sources for the characterization of semiconductor nanowires (NWs) is largely extended. Raman spectroscopy and photoluminescence (PL) are currently applied to the study of NWs. However, NWs are systems with poor thermal conductivity and poor heat dissipation, which result in unintentional heating under the excitation with a focused laser beam with microscopic size, as those usually used in microRaman and microPL experiments. On the other hand, the NWs have subwavelength diameter, which changes the optical absorption with respect to the absorption in bulk materials. Furthermore, the NW diameter is smaller than the laser beam spot, which means that the optical power absorbed by the NW depends on its position inside the laser beam spot. A detailed analysis of the interaction between a microscopic focused laser beam and semiconductor NWs is necessary for the understanding of the experiments involving laser beam excitation of NWs. We present in this work a numerical analysis of the thermal transport in Si NWs, where the heat source is the laser energy locally absorbed by the NW. This analysis takes account of the optical absorption, the thermal conductivity, the dimensions, diameter and length of the NWs, and the immersion medium. Both free standing and heat-sunk NWs are considered. Also, the temperature distribution in ensembles of NWs is discussed. This analysis intends to constitute
\end{abstract}

a tool for the understanding of the thermal phenomena induced by laser beams in semiconductor NWs.

\section{Introduction}

Semiconductor nanowires (NWs) have attracted a great deal of attention because of their potential application in electronics and optoelectronics [1-5]. Beyond the quantum confinement effects, relevant for NWs with a diameter below $20 \mathrm{~nm}[6,7]$, other physical properties are severely changed by the high aspect ratio of NWs with diameters sensibly larger than the limit for quantum effects. One of those properties is the thermal conductivity, which is significantly suppressed with respect to their bulk counterparts [8-11]. The thermal transport in NWs is a critical issue in view of their practical application, since many devices operate at high current and power densities, therefore, one needs to extract high heat power densities from the active zones of the devices [12]. On the other hand, characterization tools with submicrometer spatial resolution are necessary to boost the understanding of these structures in view of new nanodevice concepts. Laser beams constitute contactless probes for studying fundamental properties of semiconductors. PL, [13-15] optical absorption, [16-18] Raman scattering, [19$26]$ and photocurrent (PC), [27, 28] among other techniques, are currently used for the characterization of diverse semiconductor nanostructures. However, the specific geometry and dimension of the NWs blurry the interpretation of the results, because of the lack of a detailed analysis of the interaction between the focused laser beam and the NWs. The optical characterization can be carried out on either ensembles of NWs, or individual NWs. When ensembles of NWs are studied, the optical response is contributed by NWs that can have different dimensions and, therefore, a dispersion of 
their physical properties. Meanwhile, the characterization of individual NWs is challenging because of the small volume of material probed by the laser beam, which leads to the search of a compromise between the laser power necessary to detect the corresponding output signal and the heating induced by the laser beam. The NW overheating hinders the interpretation of the experimental results as reported in the literature $[12,19,21-24,29-32]$. On the other hand, the experimental approaches to measure the thermal conductivity of NWs face the difficulties of measuring the temperature at the nanometer scale, and the poor heat flow between the NW and the measurement resistors in thermal bridges. In this context, the use of optical beams as contactless thermal probes appears as an attractive approach for the measurement of the thermal conductivity of NWs, making use of the temperature dependence of certain optical properties, which can be used as local thermometers [12,33-37]. One of the critical issues when using laser beams as probe vehicles for NWs characterization is purely geometric, because the diameters of the NWs are substantially smaller than the laser beam diameter used for electro-optical characterization experiments, e.g., Raman, PL, PC. Many of these experiments are carried out with optical microscopes and laser beams focused by high magnification objectives, which results in spatially nonuniform laser power at focus, but it presents a Gaussian power profile, therefore, the excitation conditions are expected to be strongly dependent on the position of the NWs inside the laser beam spot. The use of ensembles of NWs permits to increase the output optical signal, either Raman, absorption, or luminescence; however, as mentioned above, the situation of the NWs inside the laser beam spot, determines the excitation conditions for each of them and, therefore, their optical output signal, as well as the heating induced by the laser beam should depend on the distribution of the NWs inside the laser beam spot. In this context, an exhaustive study of the interaction of the NWs with the laser beam, taking account of the energy transferred to the NWs, is still lacking; however, it appears necessary for the unambiguous interpretation of the experiments in which NWs are excited with laser beams. We present in this work a numerical study of the interaction between the Si NWs and laser beams focused by microscope lenses as used in microRaman, microPL, PC experiments. This approach can be extended to NWs of different semiconductors without loss of generality. The analysis is carried out by the solution of the Fourier heat equation inside a NW locally heated by the laser beam. The different factors that determine the NW temperature: laser wavelength and power, optical absorption, dimensions of the NWs, the thermal conductivity of the NWs, the immersion media, and the position of the NW inside the laser beam spot are taken into account. First, the behavior of individual NWs has been simulated, paying attention to the existence or not of heat sinks; afterward, the simulation has been extended to ensembles of NWs, in which several of them are simultaneously excited by the laser beam, taking account of the positions that they occupy inside the laser beam, which permits to understand the meaning of the inhomogeneous temperature distribution inside the excited volume when probing ensembles of NWs.

\section{Modeling the transport of the heat generated by the laser beam in a NW}

A laser beam striking a NW acts as a local heat source. How the NW is overheated depends on different factors: the effective power absorbed by the NW, the thermal conductivity of the NW, and the heat dissipation conditions, which are determined by the thermal contact between the NW and the supporting substrate, and the immersion medium. In terms of thermal transport, all these aspects need to be considered if one wants to give an accurate description of the interaction between the laser beam and the NW, and consequently a correct interpretation of the data acquired under laser excitation. The study is carried out by finite element methods (FEM), solving the heat transfer equation, under a local heat source represented by the laser beam power supplied at the impact region.

$\nabla \cdot(-\kappa \nabla T)=Q$

where $\kappa$ is the thermal conductivity of the NW. On the righthand side of the heat equation appears the power density in the NW, labeled $Q$. This term depends on the external heat source, in our case it is determined by the absorption coefficient, the reflectivity, and the geometry of the NW. The heat source acquires the shape of the focused laser beam itself, which presents a Gaussian power profile.

$I=I_{0} \exp \left[-2(r / w(\lambda))^{2}\right]$

where $w(\lambda)$ takes account of the radius of the laser beam spot and is defined as the distance from the beam axis where the optical intensity drops to $1 / e^{2}$, which depends on the laser wavelength. The optical absorption by NWs is a critical issue for the calculation of the laser induced heating. Because the NW diameter is smaller than the laser wavelength, the power absorbed by the NWs is diameter dependent [18, $35,38,39]$. As a consequence of this, significant differences with the bulk absorption take place. The laser absorption is calculated by solving the Maxwell equations for the interaction laser/NW. Regarding the thermal conductivity, a significant suppression has been reported with respect to the bulk values. Unfortunately, the available experimental data for NWs are scarce, and limited to a few NW diameters and a reduced temperature range $[2,8]$. The estimation of both, the optical absorption and the thermal conductivity is, therefore, a step forward to the solution of the interaction of the 
laser beam with the NWs. When solving the heat equation, one assumes a steady state behavior, with natural convective/radiative heat exchange between the NWs and the immersion medium. Convection cooling between the boundaries that interface with the cooling fluid can be described for a regular geometry in terms of a heat transfer coefficient, $\mathrm{h}$, and a temperature difference across a fictitious thermal boundary layer, given the Neumann boundary condition described as [40]:

$-\mathbf{n} .(-\kappa \nabla T)=h\left(T_{0}-T\right)+\varepsilon \sigma\left(T_{0}^{4}-T^{4}\right)$

where, $\varepsilon$ and $\sigma$ are the emissivity and the Stefan-Boltzmann constant, $T_{0}$ is the temperature of the dissipative immersion medium, and $\mathrm{h}$ is the heat transfer coefficient. The heat transfer coefficient, $h$, depends on the immersion fluid; for more details, see [40]. In NWs with thermal contact to the substrate, Neumann, for the free surfaces, and Dirichlet, for the contacting surfaces, boundary conditions were used.

\section{The absorption efficiency of NWs}

The effective laser energy absorption in bulk semiconductors depends on the laser wavelength. In the case of NWs, the dielectric mismatch between the NW and the surrounding media, plus the subwavelength dimension of the NWs, make the problem of the energy absorption by the NWs more complex than for their bulk counterparts. The solution of the Maxwell equations for the interaction of the electromagnetic field associated with the laser beam, and the $\mathrm{NW}$, results in diameter dependent absorption energy showing a series of resonances when the wavelength matches an electromagnetic eigenmode of the NW [17, 35, 38]. On the other hand, the absorption can be modified according to the distance between the NW and the supporting substrate, e.g., in suspended NWs, one should consider such a distance [34]; furthermore, the absorption energy is also changed by the immersion medium. The absorption by the NW can be represented more readily through the absorption efficiency, Qa, defined in the frame of Mie theory as a dimensionless magnitude, corresponding to the ratio between the absorption cross section and the projected area of the NW [42]. It is possible calculating the absorption efficiency using Mie solutions of the Maxwell equations, allowing us to handle the true energy absorbed for the analysis of the interaction laser/NW [17]. The accuracy of this solution of Maxwell's equations has been demonstrated in [35], thus justifying the evaluation of the Qa according to $[17,35,42]$. The absorption efficiencies for green $\operatorname{Ar}(514.5 \mathrm{~nm})$ and red helium neon $(632.8 \mathrm{~nm})$ laser lines are represented in Fig. 1 as a function of the Si NW diameter, showing the sharp resonances of the laser energy absorbed for specific diameters. The resonances change with the laser wavelength and

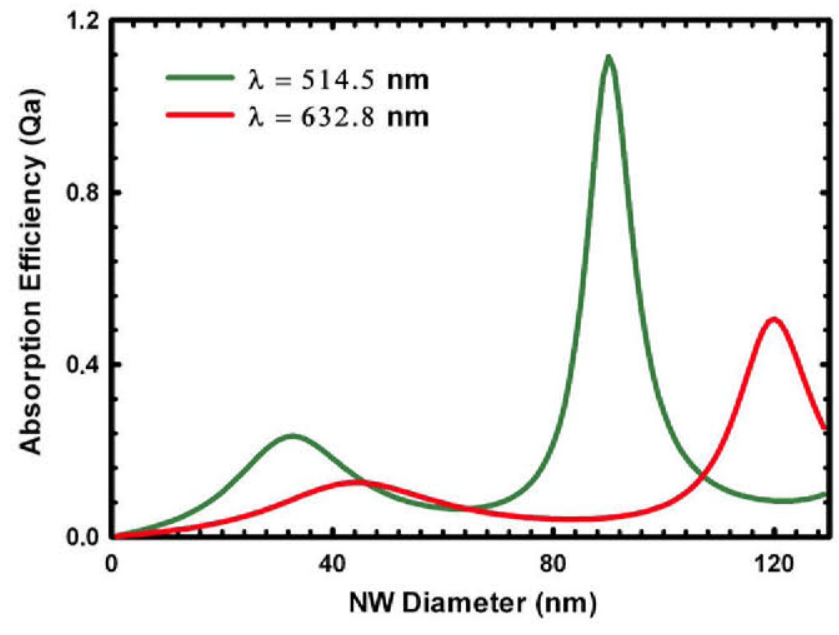

Fig. 1 Absorption efficiency vs. NW diameter of silicon NWs calculated for two laser wavelengths, $632.8 \mathrm{~nm}$ and $514.5 \mathrm{~nm}$. Note the occurrence of resonances for some diameters, depending on the laser wavelength

the immersion medium, and the simulations of Fig. 1 correspond to NWs immersed in air. The optical functions used for the calculation were taken from [41]. These resonances have to be considered when estimating the heat transferred to the NW by the absorption of the laser beam.

\section{The thermal conductivity of NWs}

The thermal conductivity of semiconductor NWs is a relevant parameter, since it has been demonstrated to be substantially suppressed with respect to the bulk value [2, 8]. Experimentally measured thermal conductivity data of NWs are available for a few diameters at temperatures usually below $320 \mathrm{~K}[8,10,11,36,43,45]$. However, the NWs easily reach temperatures well above room temperature under the excitation with a focused laser beam; therefore, values of the thermal conductivity at temperatures over $320 \mathrm{~K}$ are necessary for solving the heat transfer in NWs. The limitation of the thermal conductivity of NWs is due to the phonon boundary scattering, therefore, the thermal conductivity is decreased when reducing the NW diameter, but also for rough surface NWs [9, 44, 46, 47]. Keeping all these considerations in mind, we have proceeded to calculate the thermal conductivity of Si NWs at temperatures above $320 \mathrm{~K}$. For this, we have used a modified Callaway-Holland formalism [48], and we checked the model by the satisfactory fitting to the available experimental data on both smooth and rough NWs; for more details, see Anaya et al. [48]. This model for the thermal conductivity yields a good response for bulk semiconductors all over the full temperature range. Therefore, we have assumed that the calculated thermal conductivities provide a good approximation to the thermal conductivity of NWs in 


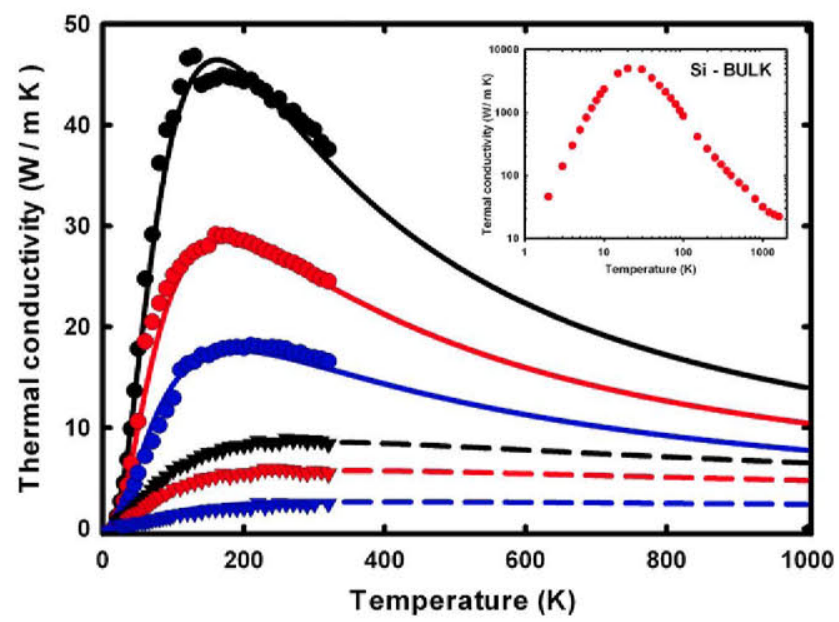

Fig. 2 Thermal conductivity vs. $T$ calculated according to [48] The symbols correspond to the experimental values reported in [2] (triangles, rough NWs with diameters of 115, 98 and $50 \mathrm{~nm}$ ) and [8] (circles, smooth NWs with diameters of 115,56 and $37 \mathrm{~nm}$ ). The bulk Si values are displayed in order to get a better picture of the reduction in thermal conductivity of the NWs

an extended temperature range above $300 \mathrm{~K}$, where experimental data are lacking. The results of our estimations for thermal conductivities of both smooth and rough NWs are shown in Fig. 2, where one can see how the model used fits very satisfactorily to the experimental data for the thermal conductivity of smooth and rough Si NWs reported on $[2,8]$.

\section{Influence of the laser wavelength and power, and the surrounding dissipative media}

We studied separately the influence of the different factors contributing to the temperature reached by the NW under the excitation with the focused laser beam. We first calculated the temperature reached by a free standing Si NW $(37 \mathrm{~nm}$ diameter, and $5 \mu \mathrm{m}$ long) in two different dissipative immersion media, air and water, for two laser wavelengths (514.5, and $632.8 \mathrm{~nm}$, respectively), as a function of the incident laser power. We selected such diameter because is one of the diameters for which experimental data about the thermal conductivity are available $[2,8]$. The temperature plotted in Fig. 3 corresponds to the temperature reached by the NW in the zone of the laser beam impact, being located this point at the center of the NW. Significant temperature rise is already observed at low laser powers of $10-15 \mu \mathrm{W}$, which suggests that even at low laser power the corresponding output signals, either Raman, PL, or PC, might be blurred by heating. One also observes a clear dependence with the excitation wavelength, as the total amount of energy absorbed by the NW depends on the wavelength, though, as shown in the paragraph dealing with the absorption efficiency of NWs, it

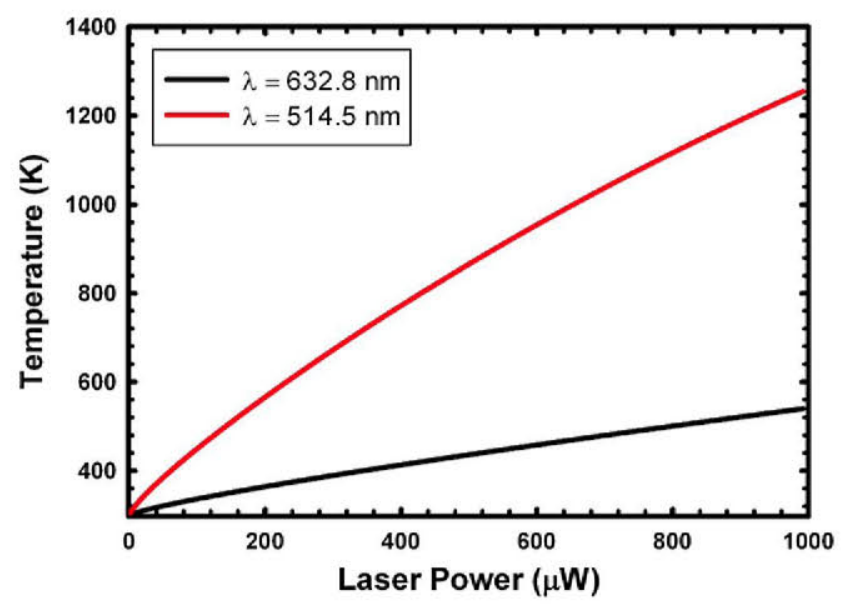

Fig. 3 Temperature vs. laser power calculated for a free standing NW of $37 \mathrm{~nm}$ diameter and $5 \mu \mathrm{m}$ length, for $514.5 \mathrm{~nm}$, and $632.8 \mathrm{~nm}$ excitation wavelengths, in air. The laser impact on the center of the NW

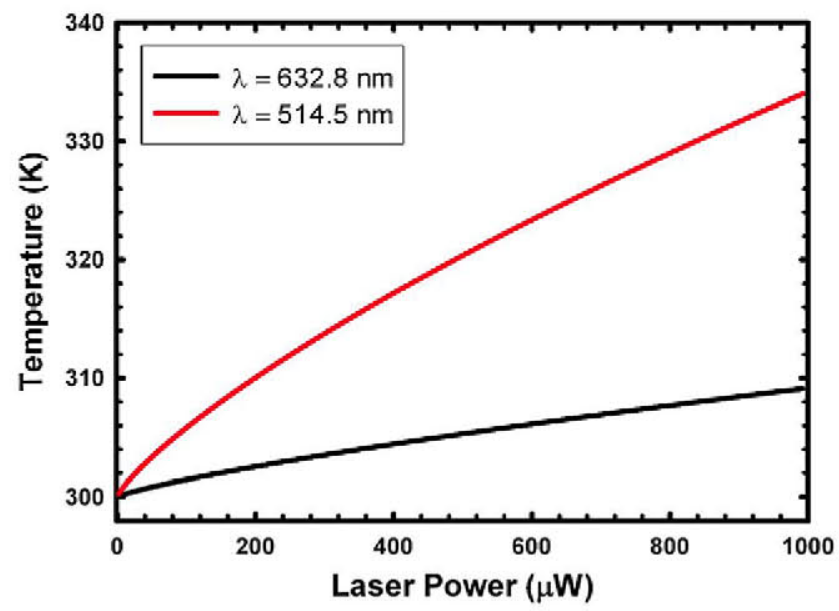

Fig. 4 The same as Fig. 3 but with the NW immersed in water

does not obey a simple absorption relation as in bulk materials, because of the existence of the absorption resonances, Fig. 1.

The importance of the dissipative medium is revealed in Fig. 4, where one represents the temperature reached by the same NW assuming similar absorption efficiency; the temperature is dramatically lowered when the NW is immersed in water, a medium with higher thermal conductivity, and principally much more dense than air, which substantially enhances the heat transfer coefficient, $h$ in Eq. (3) [40]; note that the immersion medium can also influence the absorption efficiency; therefore, the laser power along the abscissa of Fig. 4 is the effective power absorbed by the NW in each case, which permits to isolate the role of the immersion medium only in terms of heat dissipation. The role of the dissipative media on the shape of the Raman spectrum of $\mathrm{Si}$ NWs has been evidenced in $[19,24]$. 


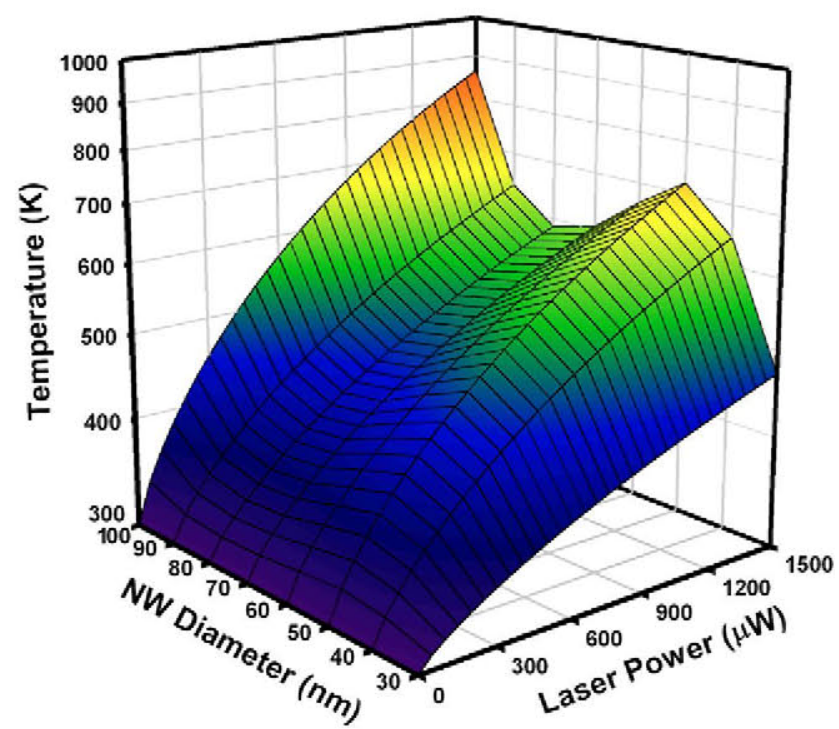

Fig. 5 3D plot representing the temperature in a free standing NW as a function of laser power, and NW diameter. The temperature for the different laser powers follows the absorption resonances. The simulation is done for $5 \mu \mathrm{m}$ long NWs, laser wavelength $632.8 \mathrm{~nm}$, and laser impact on the center of the NW

\section{Influence of the NW dimensions}

The dimensions of the NW, both diameter and length, play a relevant role on the NW heating. As shown previously, the NW diameter determines the physical parameters, such as the absorption efficiency, and the thermal conductivity. Once the laser impacts on the NW, the heat is distributed over the NW reaching the equilibrium between the absorbed and dissipated energy. Therefore, the temperature reached by the NW is also tributary of the NW dimension. The laser induced heating of a free standing NW as a function of the NW diameter and length is summarized in Figs. 5 and 6, respectively. In both cases, the NW was in the center of the laser beam spot. The temperature reached by the NW under the laser beam excitation is the consequence of the reduced thermal conductivity, the poor heat dissipation, and the different laser energy absorbed, both because of the absorption volume, but also because of the diameter dependence of the optical absorption efficiency, Qa, Fig. 2. Figure 5 shows the temperature reached by the NW as a function of its diameter and the laser power; one observes that the temperature of the NWs does not follow a simple dependence with the NW diameter, but it is modulated by the Qa. Regarding the NW length, one observes that for NWs with the same diameter the temperature decreases as the length increases, because the larger the surface and volume, the better the heat dissipation. Under the excitation, conditions used for this simulation (laser power at focus of $200 \mu \mathrm{W}$ ), while short NWs have a poor radiative-convective dissipation and can reach higher temperature; the temperature reached by the longer

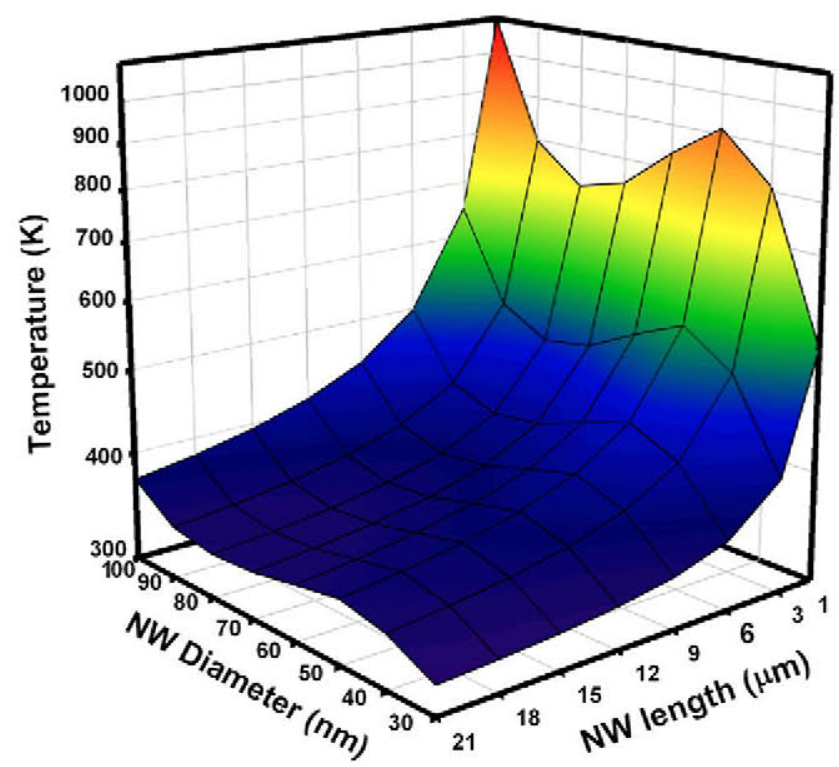

Fig. $63 \mathrm{D}$ plot representing the temperature in free standing NWs as a function of the NW diameter and length; the temperature follows the absorption resonances. It decreases with increasing the NW length because of the higher dissipation when the NW volume increases. The simulation is done, laser wavelength $514.5 \mathrm{~nm}, 200 \mu \mathrm{W}$ power, and laser impact on the center of the NW

NWs can be hundreds of degrees lower than the one reached by short NWs under those excitation conditions. Note that, due to the large increase in volume and increased dissipation surface, the longer the NW the lower the influence of the diameter.

\section{NWs with heat sinks}

The situation changes in the case of a NW anchored to the substrate, or suspended NWs with the ends linked to a substrate, which is a common configuration for electronic devices as is the case of NWs with two electric contacts, or NWs mounted on a thermal bridge for thermal conductivity measurements. Under these experimental assemblies, the heat transfer is carried out not only by the convective/radiative (Neumann conditions) heat exchange through the lateral surface of the NWs, similar to the free standing NWs, but also by conduction heat exchange either through the base of the NW linked to the substrate, or the side contact with the heat sink (Fig. 7a) (Dirichlet boundary conditions). The convective/radiative heat exchange plays a less relevant role as compared to the thermal transport through the heatsinks. In this situation, the thermal resistance between the NW and the heatsink is critical. This is one of the main difficulties when using thermal bridges for measuring the thermal conductivity of the NWs, because the thermal resistance at the contact parts can blurry the data. Figure $7 \mathrm{~b}$ 


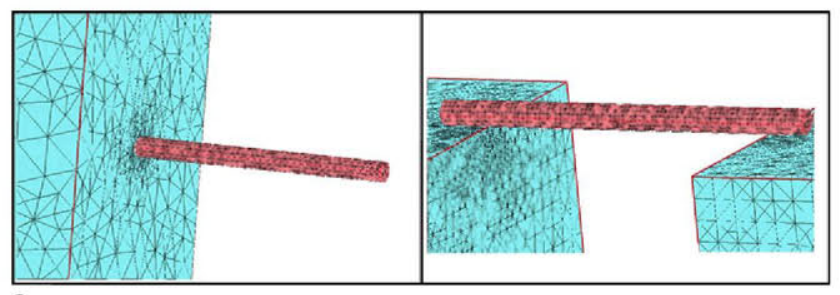

a

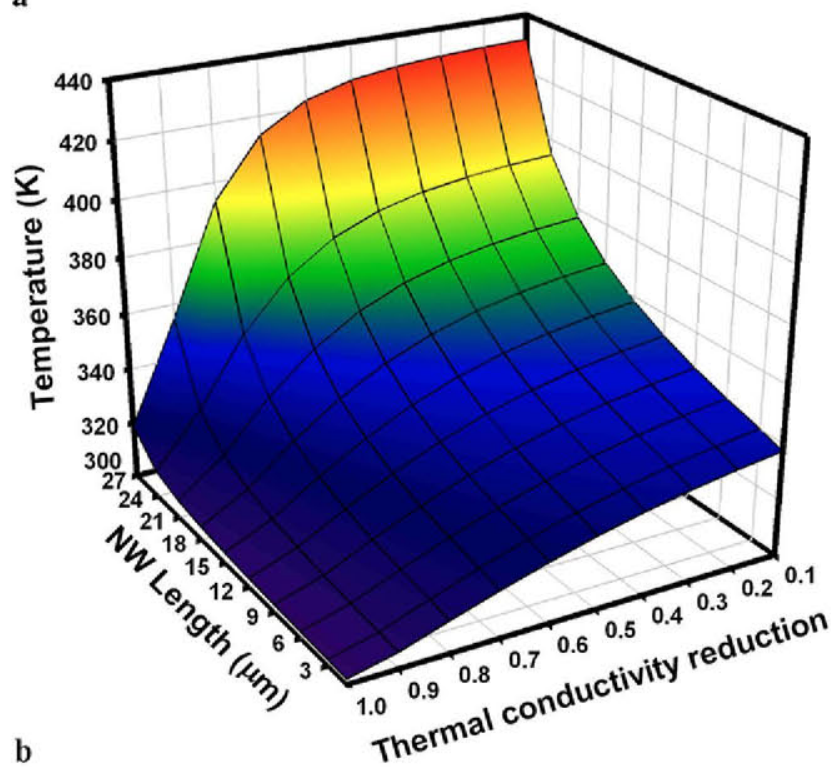

Fig. 7 (a) Scheme of NWs linked to the substrate by one of the ends, and suspended NWs in two metallic pads. (b) Temperature vs. NW length and thermal conductivity for a NW linked to the substrate by one of the ends considered as an ideal heatsink (Dirichlet boundary condition). One observes the paramount role played in this case by the thermal conductivity of the NWs; the temperature is enhanced by the combination of low thermal conductivity and long NWs. The NWs are $37 \mathrm{~nm}$ diameter, the laser wavelength 514.5 and $200 \mu \mathrm{W}$ power. The laser impacts in the center of the NWs

shows the temperature reached by a $37 \mathrm{~nm}$ diameter NW anchored to the substrate by one of the ends, as a function of the NW length and the thermal conductivity; the substrate temperature is settled up at $300 \mathrm{~K}$ constituting the boundary condition. Note that in a configuration of this type, the thermal transport to the heatsink is dominated by the thermal conductivity, a property, which for a given NW diameter, can take very different values depending on the characteristics of the NW surface. One observes that short NWs reach lower temperature than long NWs, and that the reduction of the thermal conductivity enhances the NW temperature, especially for long NWs, which constitutes an opposite behavior with respect to the free standing NWs; in fact, in free standing NWs the temperature reached by the NW depends on the NW dimensions and the heat dissipation through the immersion medium, while in the NWs with heatsinks, the heat transport across the NW becomes the relevant dissipation mechanism. Because of the thermal conduction through the base of the NW toward the substrate the short NWs accumu- late less heat than long NWs and, therefore, the temperature reached is lower than for long NWs. Anyway, the temperatures calculated are much lower than the corresponding temperatures calculated for the equivalent free standing NWs. Nevertheless, the experimental observations point to higher temperatures than those calculated here [24], which suggests the existence of thermal boundary resistances (TBR) at the interface between the NW and the substrate [49]. Actually, we have just considered that the interface between the NW and the substrate is free of TBR.

\section{Position of the NW inside the beam spot}

Before discussing the temperature distribution in suspended NWs, we will discuss the influence of the position of the NW with respect to the laser beam axis. As mentioned above, the NW diameter is generally more than one order of magnitude smaller than the laser beam spot diameter; this point presents a high relevance for the characterization of NWs with laser beams. Because of the Gaussian distribution of the laser power, the energy absorbed by the NW must strongly depend on the position of the NW inside the focused laser beam spot. This is the reason why we do not use the common term of the incident laser power density; it does not make sense in the case of objects much smaller than the laser beam, which has a Gaussian power profile. On the other hand, one should note that the NWs diameters are below the diffraction limit; therefore, they cannot be resolved in the optic microscope, which makes difficult to achieve a perfect alignment of the NW and the laser beam. Therefore, the study of the influence of the position of the NW with respect to the laser beam axis is essential to understand the temperature increase of the NWs under the excitation with the laser beam. The temperature reached by the NW when the laser line scan crosses transversally the NW is summarized in Fig. 8. One observes large temperature differences in the NW temperature according to its relative position with respect to the laser beam. This suggests that it is difficult to compare measurements on NWs, which the positions inside the laser beam are not equivalent, which is the usual situation when exciting ensembles of NWs with the laser beam.

\section{Temperature distribution inside the NWs}

A very important point concerns the temperature distribution inside the NW when excited with the laser beam. The temperature gradients are determined by the thermal conductivity and the heat sinks. One has to distinguish between the temperature gradients generated over the full $\mathrm{NW}$, and the local temperature gradients restricted to the region of 
the NW under laser illumination, which is the region of interest for optical measurements. While the temperature distribution over the full NW is useful for the estimation of the thermal conductivity of the NWs, the second gradient is crucial for the understanding of the spectroscopic data under study. For example, the Raman signal is exclusively generated at the region of the NW where the laser beam impacts. This very local gradient has been assumed by some authors in the order of a few hundred $K / \mu \mathrm{m}$ [22], however, there is not evidence that could support this assertion, other than the anomalous large Raman band measured in bundles of NWs. We have estimated the temperature distribution inside the NW, varying the thermal conductivity down to the small-

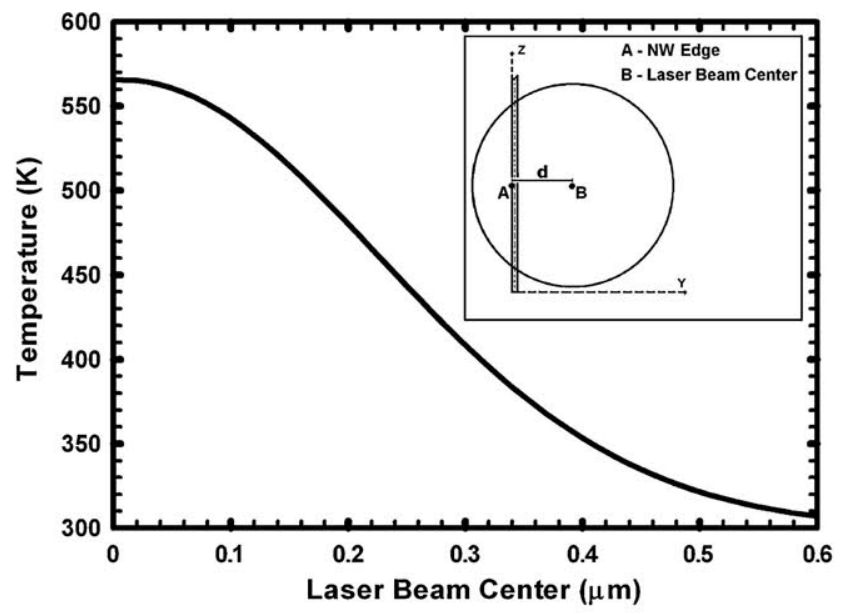

Fig. 8 Temperature in the center of the NW ( $37 \mathrm{~nm}$ diameter and $5 \mu \mathrm{m}$ long) as a function of the position of the NW with respect to the axis of the laser beam spot (see the inset). The laser wavelength was $514.5 \mathrm{~nm}$, the laser power $200 \mu \mathrm{W}$, and the laser beam diameter was $1 \mu \mathrm{m}$

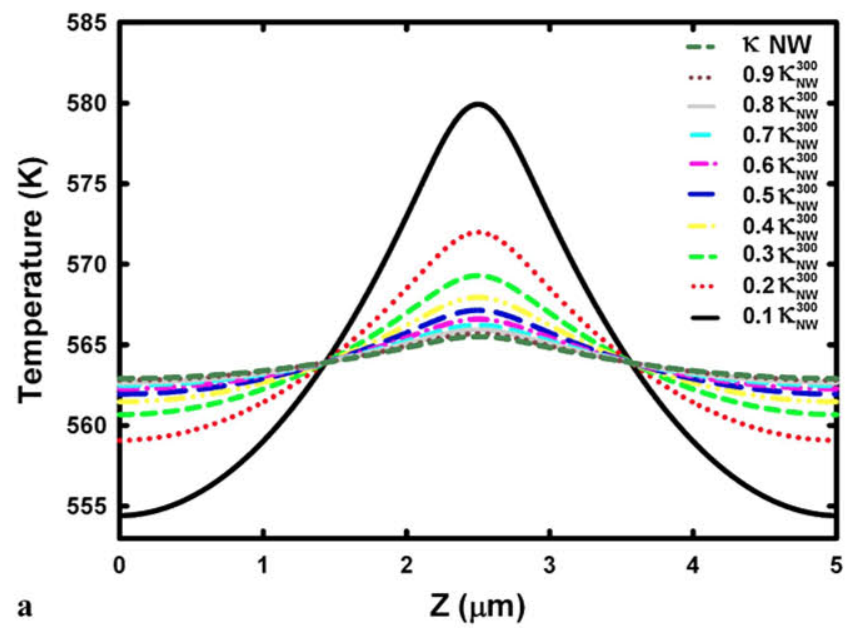

Fig. 9 (a) Temperature distribution along a free standing NW (37 nm diameter and $5 \mu \mathrm{m}$ long) with different values of the thermal conductivity, ranging from the estimated one for a smooth NW to the lowest thermal conductivity estimated for very rough NWs. The laser beam $(200 \mu \mathrm{W}$ and $514.5 \mathrm{~nm}$ ) impacts on the center of the NW. (b) Temper- est values reported for rough NWs [2]. Figure 9a shows the temperature profiles calculated as a function of the thermal conductivity for a free standing NW with the laser beam impacting on the center of the NW. The case of a NW with two heatsinks at the two ends of the NW is illustrated in Fig. 9b. The relevant result is that the temperature gradient at the laser beam diameter scale is only a few degrees, even for the NWs with the lowest thermal conductivity; therefore, in view of those results, one can argue that the temperature in the illuminated volume is roughly homogeneous, and one cannot expect large temperature gradients at such dimension scale, $\approx 1 \mu \mathrm{m}$.

\section{Suspended NWs}

The ideal configuration for measuring thermal conductivities using thermal bridges, and for most of the electronic devices, consists of NWs suspended in between two metallic resistor/electrode pads. This configuration has been used for measuring the thermal conductivity of NWs [12, 33-37]. The temperature distribution along the suspended part of a one-dimensional structure under a local laser beam excitation, which the position is scanned along the NW, was deduced by Hsu et al. [33], as to present a parabolic form according to the following expression:

$\Delta T(x)=-\frac{P}{\kappa A}\left(\frac{x^{2}}{L}+\frac{L x}{4}\right)$

This approach has subsequently been used for semiconductor NWs [34, 37], where $P$ is the power absorbed by the NW, $L$ the length of the suspended part, $A$ the cross section

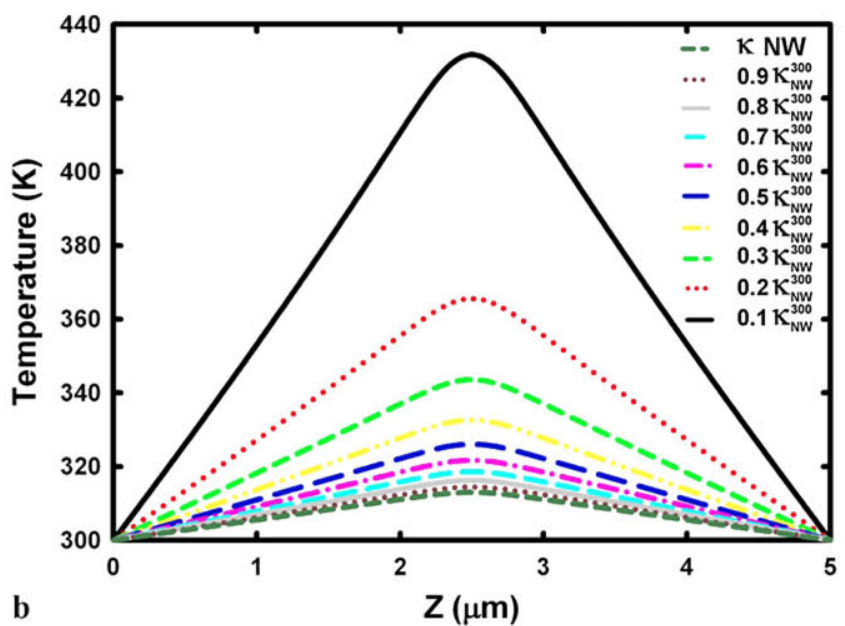

ature distribution along the NWs simulated in (a) but with two ideal heatsinks at the ends. The laser beam is here $500 \mu \mathrm{W}$ with respect to the $200 \mu \mathrm{W}$ for free standing NW of (a). Note in both figures the small temperature gradient at the scale of the laser beam spot 


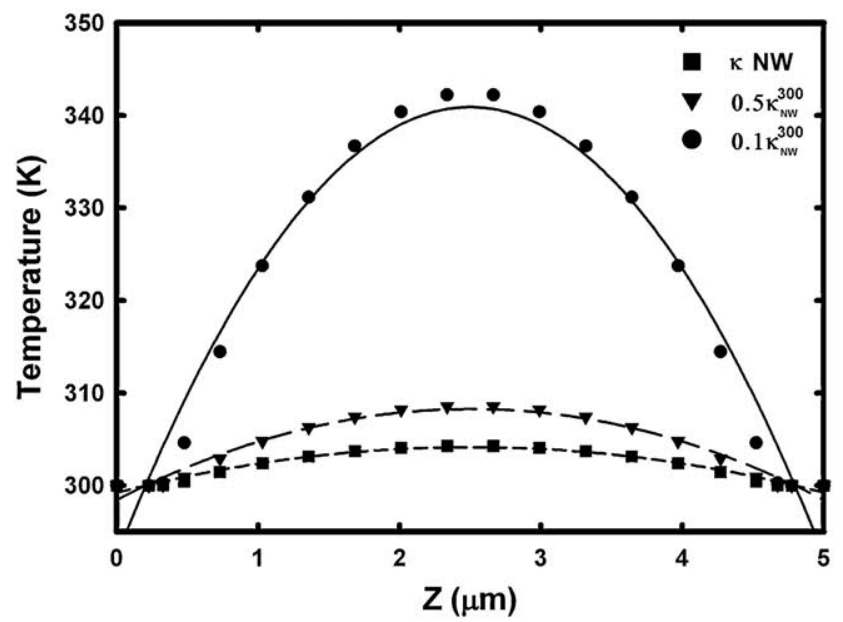

Fig. 10 Temperature as a function of the laser beam position along a suspended NW with two ideal heatsinks at the ends (symbols). The calculation is done for three different values of the thermal conductivity and a laser power of $200 \mu \mathrm{W}(514.5 \mathrm{~nm})$. The lines are the fits to the parabola distribution described by Eq. (4) [33]

of the NW, $\kappa$ the thermal conductivity, and $x$ the coordinate of laser impact. For the estimation of the absorbed power, one needs to calculate the absorption efficiency, and also one needs to know the position of the NW with respect to the laser beam according to our previous considerations. By calculating the temperature reached by the suspended part of the NW when the laser beam is scanned along the NW, following a scan line perfectly aligned with the NW, one obtains the parabola estimated by Hsu et al. [33], which is an excellent validation of our procedure. The shape of the parabola depends on intrinsic factors as the thermal conductivity of the NW, and on extrinsic factors as the size of the NW and the thermal resistance of the contact between the NW ends and the supporting substrate. Figure 10 shows the results of the simulation for different thermal conductivities for a suspended NW with dimensions of $5 \mu \mathrm{m}$ in length and a diameter of $37 \mathrm{~nm}$, with $4.5 \mu \mathrm{m}$ in the suspended part of the NW [48], which agree with the parabola predicted by Hsu et al. [33].

\section{Ensembles of NWs}

The above results are relevant to the optical measurements on ensembles of NWs. In fact, when several NWs are being simultaneously probed by the laser beam, each of them are not excited under the same conditions, but the excitation conditions for each NW depend on their relative position with respect to the laser beam axis, and also on their own diameters, and the position over the length of the NW where the laser beam impacts; all these factors are sources of dispersion, which must be reflected in the temperatures reached by the different NWs. Besides, the strong dependence of the NWs properties with the dimension can also contribute to reach different temperatures between the NWs simultaneously excited by the laser beam. The consequence of this is a very inhomogeneous temperature distribution inside the volume excited by the laser beam; but not because of the large temperature gradients inside the NWs, which we have shown that are almost negligible, but because of the very different temperatures that can be reached by the NWs, depending on their dimensions, and position inside the laser beam spot. To illustrate this, the temperature reached by four identical NWs aligned parallel, for geometric simplicity, and probed by the laser beam was calculated for different positions of the laser beam with respect to the NWs. One observes significant temperature differences between the four NWs depending on their relative positions, and also significant changes in the temperature as the laser spot is scanned across the four NWs, Fig. 11; e.g., depending on the position of the laser beam with respect to the NWs they can reach maximum temperature of $550 \mathrm{~K}$ and minimum of $335 \mathrm{~K}$, which are transformed to $470 \mathrm{~K}$ and $380 \mathrm{~K}$ when the laser beam is moved only by $0.1 \mu \mathrm{m}$, across the bundle of NWs. This clearly evidences that the volume under excitation is very inhomogeneous, in the sense that the NWs are excited under different incident powers, which should be reflected in the temperature of each NW; this means that the optical output signal (Raman, PL, etc.) of bundles of NWs under the laser excitation, can present an almost unsolvable dispersion. This example illustrates how the excitation of bundles of NWs by a laser beam focused through an optical microscope results in very inhomogeneous excitation conditions for the different NWs composing the population under excitation, which necessarily blurs the interpretation of the output optical signal data extracted from the measurement. We have here focused only on the thermal aspects of the interaction between the laser beam and the NWs; nevertheless the difference in the excitation conditions can also affect other properties beyond the purely thermal aspect.

\section{Conclusions}

Focused laser beams impacting semiconductor NWs constitute local heat sources that enhance the NW temperature. The analysis of this effect has been carried out using finite element methods (FEM) for solving the heat transfer equation inside the NW. The temperature distribution inside the NWs is demonstrated to be dependent on intrinsic factors as the thermal conductivity of the NWs and extrinsic factors, as the dimension of the NWs, immersion media, and excitation configuration. It is demonstrated that high temperatures can be reached in NWs excited with moderate laser powers, as those used in typical microRaman or microPL experiments. 


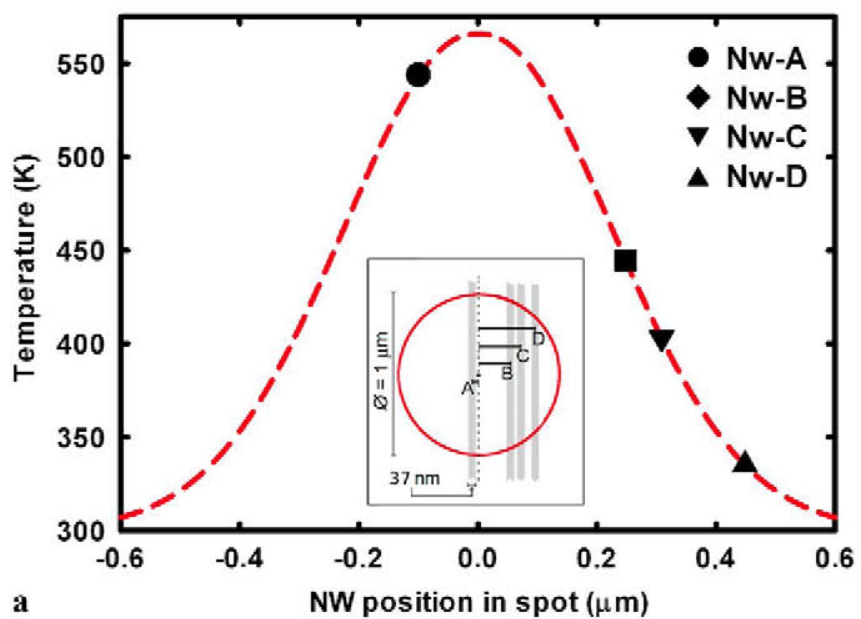

Fig. 11 Temperatures calculated for an ensemble of four identical $\mathrm{NWs}$ (37 nm diameter and $5 \mu \mathrm{m}$ long) occupying different positions inside the laser beam spot $(514.5 \mathrm{~nm}, 200 \mu \mathrm{W})$. Two NWs configura-

Significant differences are revealed between the behavior of free standing and heatsink NWs. A very important aspect concerns the temperature gradients at the scale of the laser beam, which are demonstrated to be very small, even for the most unfavorable case of very rough NWs with strongly suppressed thermal conductivity. Finally, one reveals that ensembles of NWs excited with the laser beam constitute very inhomogeneous media, which suggests that the measurements carried out with ensembles of NWs excited by focused laser beams are strongly conditioned by such a lack of homogeneity. An application of this analysis to the microRaman spectroscopy of Si NWs will be the subject of a forthcoming work.

Acknowledgements This work was funded by the Spanish Government (MAT-2007-66181-C03 and MAT-2010-20441-C02) and by Junta de Castilla y León (VA051A06 -GR202).

\section{References}

1. Y. Cui, C.M. Lieber, Science 291, 851 (2001)

2. A.I. Hochbaum, R. Chen, R.D. Delgado, W. Liang, E.C. Garnett, M. Najarian, A. Majumdar, P. Yang, Nature 451, 163 (2008)

3. B.M. Kayes, H.A. Atwater, N.S. Lewis, J. Appl. Phys. 97, 114302 (2005)

4. Y. Nakayama, P.J. Pauzauskie, A. Radenovic, R.M. Onorato, R.J. Saykally, J. Liphardt, P. Yang, Nature 447, 1098-1101 (2007)

5. N. Singh, K.D. Buddharaju, S.K. Manhas, A. Agarwal, S.C. Rustagi, G.Q. Lo, N. Balasubramanian, D.L. Kwong, IEEE Trans. Electron Devices 55, 3107 (2008)

6. H. Richter, Z.P. Wang, L. Ley, Solid State Commun. 39, 625 (1981)

7. I.H. Campbell, P.M. Fauchet, Solid State Commun. 58, 739 (1986)

8. D. Li, Y. Wu, P. Kim, L. Shi, P. Yang, A. Majumdar, Appl. Phys. Lett. 83, 2934 (2003)

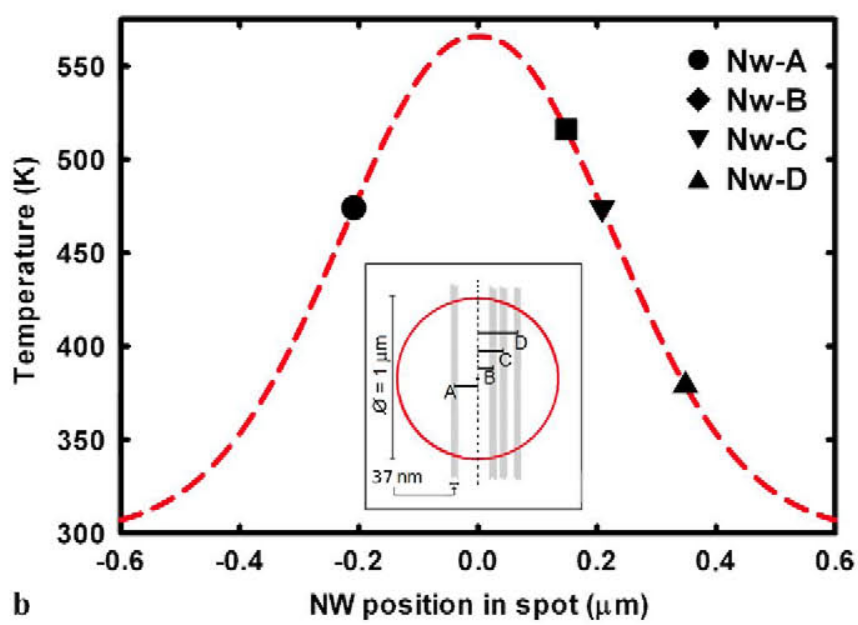

tions are shown, (a) and (b), showing the very different temperatures that can be reached by the NWs

9. Y. Park, J. Kim, H. Kim, I. Kim, K. Lee, D. Seo, H. Choi, W. Kim, Appl. Phys. A 104, 7 (2011)

10. C. Guthy, C. Nam, J.E. Fischer, J. Appl. Phys. 103, 064319 (2008)

11. C. You, Nano Lett. 12, 2918 (2012)

12. T. Westover, R. Jones, J.Y. Huang, G. Wang, E. Lai, A.A. Talin, Nano Lett. 9, 257 (2009)

13. D.D.D. Ma, S.T. Lee, J. Shinar, Appl. Phys. Lett. 87, 033107 (2005)

14. F. Martelli, M. Piccin, G. Bais, F. Jabeen, S. Ambrosini, S. Rubini, A. Franciosi, Nanotechnology 18, 125603 (2007)

15. O. Demichel, F. Oehler, V. Calvo, P. Noé, N. Pauc, P. Gentile, P. Ferret, T. Baron, N. Magnea, Physica E 41, 963 (2009)

16. X.Q. Xie, W.F. Liu, J.I. Oh, W.Z. Shen, Appl. Phys. Lett. 99, 033107 (2011)

17. L. Cao, J.S. White, J.S. Park, J.A. Schuller, B.M. Clemens, M.L. Brongersma, Nat. Mater. 8, 643 (2009)

18. G. Brönstrup, N. Jahr, C. Leiterer, A. Csaki, W. Fritzsche, S. Chistiansen, ACS Nano 4, 7113 (2010)

19. H. Scheel, S. Reich, A.C. Ferrari, M. Cantoro, A. Colli, C. Thomsen, Appl. Phys. Lett. 88, 233114 (2006)

20. S. Bhattacharya, S. Samui, Appl. Phys. Lett. 84, 1564 (2004)

21. S.R. Gupta, Q. Xiong, C.K. Adu, U.J. Kim, P.C. Eklund, Nano Lett. 3, 627 (2003)

22. K.W. Adu, H.R. Gutiérrez, U.J. Kim, P.C. Eklund, Phys. Rev. B 73, 155333 (2006)

23. G.W. Zhou, Z. Zhang, D.P. Yu, Appl. Phys. Lett. 73, 677 (1998)

24. A. Torres, A. Martín-Martín, O. Martínez, A.C. Prieto, V. Hortelano, J. Jiménez, A. Rodríguez, J. Sangrador, T. Rodríguez, Appl. Phys. Lett. 96, 011904 (2010)

25. L. Zhang, W. Ding, Y. Yan, J. Qu, B. Li, L.-Y. Li, K.T. Yue, D.P. Yu, Appl. Phys. Lett. 81, 4446 (2002)

26. I. Zardo, G. Abstreiter, A. Fontcuberta, in Nanowires, ed. by P. Prete (INTECH, Rijeka, 2010), p. 227. ISBN978-953-7619-79-4, Chap. 12

27. Y. Ahn, J. Dunning, J. Park, Nano Lett. 5, 1367 (2005)

28. H.Y. Chen, R.S. Chen, N.K. Rajan, F.C. Chang, L.C. Chen, K.H. Chen, Y.J. Yang, M.A. Reed, Phys. Rev. B 84, 205443 (2011)

29. R. Jalilian, G.U. Sumanasekera, H. Chandrasekharan, M.K. Sunkara, Phys. Rev. B 74, 155421 (2006)

30. G.S. Doerk, C. Carraro, R. Maboudian, Phys. Rev. B 80, 073306 (2009)

31. E. Alarcón-Lladó, J. Ibañez, R. Cuscó, L. Artús, J.D. Prades, S. Estradé, J.R. Morante, J. Raman, Spectroscopy 42, 153 (2011) 
32. K. Roodenko, I.A. Goldthorpe, P.C. MacIntyre, Y.J. Chabal, Phys. Rev. B 82, 115210 (2010)

33. I.K. Hsu, R. Kumar, A. Bushmaker, S.B. Cronin, M.T. Pettes, Appl. Phys. Lett. 92, 063119 (2008)

34. M. Soini, I. Zardo, E. Uccelli, S. Funk, G. Koblmuller, A. Fontcuberta, G. Abstreiter, Appl. Phys. Lett. 97, 263107 (2010)

35. G.S. Doerk, C. Carraro, R. Maboudian, ACS Nano 4, 4908 (2010)

36. Y. Zhang, J. Kristofferson, A. Shakouri, D. Li, A. Majumdar, Y. Wu, R. Fan, P. Yang, IEEE Trans. Nanotechnol. 5, 67 (2006)

37. X.F. Liu, R. Wang, Y.P. Jiang, Q. Zhang, X.Y. Shan, X.H. Qiu, J. Appl. Phys. 108, 054310 (2010)

38. L. Cao, P. Fan, A.P. Vasudev, J.S. White, Z. Yu, W. Cai, J.A. Schuller, S. Fan, M.L. Brongersma, Nano Lett. 10, 439 (2010)

39. L. Cao, J. Park, P. Fan, B. Clemens, M.L. Brongersma, Nano Lett. 10, $1229(2010)$

40. J.R. Backhurst, J.H. Harker, J.F. Richardson, J.M. Coulson, Chemical Engineering, 6th edn., vol. 1 (Butterworth-Heinemann, Oxford, 1999). Chap. 9
41. B.K. Sun, X. Zhang, C.P. Grigoropoulos, Int. J. Heat Mass Transf. 40, 1591-1600 (1997)

42. M. Kerker, The Scattering of Light and Other Electromagnetic Radiation (Academic Press, New York, 1969), Chap. 3 and Chap. 8

43. H. Kim, L. Kim, H. Choi, W. Kim, Appl. Phys. Lett. 96, 233106 (2010)

44. R. Chen, A.I. Hochbaum, P. Murphy, J.E. Moore, P. Yang, A. Majumdar, Phys. Rev. Lett. 101, 105501 (2008)

45. M.C. Wingert, Z.C.Y. Chen, E. Dechaumphai, J. Moon, J. Kim, J. Xiang, R. Chen, Nano Lett. 11, 5507 (2011)

46. J. Lim, K. Hippalgaonkar, S.C. Andrews, A. Majumdar, P. Yang, Nano Lett. 12, 2475 (2012)

47. H. Kim, Y. Park, I. Kim, J. Kim, H. Choi, W. Kim, Appl. Phys. A 104, 23 (2011)

48. J. Anaya, T. Rodríguez, J. Jiménez, Nanowires, Recent Advances (INTECH, Rijeka, ISBN980-953-307-525-4, 2012 (to be published))

49. J.Y. Duquesne, Phys. Rev. B 79, 153304 (2009) 